Notas Clínicas

\title{
Un caso de melanoma maligno meníngeo en un varón adulto que debuta con paraplejia
}

I. Díaz de Corcuera Frutos, S. Arévalo Lobera, L. Basterretxea Badiola, L. Egaña Otaño, J. Mínguez Manrique,

A. Lacasta Muñoa

\section{Resumen}

Este es el caso de un varón afecto de un probable melanoma meníngeo que debuta con una paraplejia por compresión medular. El melanoma maligno de localización en el sistema nervioso central varía en las características citológicas mucho más que en otros lugares. En ocasiones puede resultar muy dificultosa e incluso imposible su precisa clasificación histológica. Paralelamente se revisará el tratamiento bioquimioterápico en este tipo de tumores.

Palabras clave:

Melanoma maligno meníngeo. Tratamiento. Interferón.

Oncología, 2004; 27 (9):553-557

Unidad de Oncología Médica

Complejo Hospitalario Donostia

Guipúzcoa 


\section{Summary}

We report the case of a man with a probable malignant melanoma. The first symptom was a paraplegia caused by spinal cord compression. Malignant melanoma of central nervous system offers more types of cytological features than in other localizations. Some times it is very difficult and even impossible to reach a precise histological classification. Taking the opportunity of this report we review the biochemotherapy approach of these kind of tumors.

Key words: Meningeal malignant melanoma. Treatament. Interferon.

\section{Introducción}

Las lesiones primarias tumorales pigmentadas de las meninges son raras. Estas pueden ser localizadas o difusas, benignas o malignas y en la mayoría de las ocasiones plantean un difícil diagnóstico diferencial por las características citológicas especiales de esta localización. Los síntomas que producen están directamente relacionados con su ubicación, por ejemplo, la compresión medular, que como en este caso puede obligar a una actuación terapéutica urgente. El tratamiento de elección para los melanomas localizados es la cirugía pero posteriormente a la resección, en función del estadio, pueden precisar de un tratamiento adyuvante que aún hoy en día está siendo evaluado. Los melanomas diseminados pueden beneficiarse de la bioquimioterapia.

\section{Caso clínico}

Presentamos el caso de un varón de 54 años sin antecedentes médicos o quirúrgicos a destacar, que refería desde marzo del 2000 notar de manera ocasional un dolor torácico intermitente que cedía espontáneamente. En marzo del 2001 dicho dolor comenzó a ser más frecuente, por lo que se inicia su estudio sin hallarse patología. En octubre de 2001 comenzó con lo que él describió como "sensación extraña" en extremidades inferiores, y fue a la semana de dicha clínica cuando en pocas horas perdió la movilidad completa de extremidades inferiores y sensibilidad desde región submamilar.

Acudió al Servicio de Urgencias del Hospital de Mendaro donde se realizó un análisis, una radiografía (Rx) de tórax urgente, y un EKG con resultados dentro de la normalidad pero con una clínica de paraparesia subaguda sugestiva de lesión medular por lo que fue trasladado al Hospital de Aránzazu (3-10-2001). En la exploración física llevada a cabo en este servicio se observó también una paraparesia de EID $3 / 5$ y en Ell de 0-1/5. La sensibilidad también se vió afectada detectándose una hipoestesia inferior a D9-10 con exaltación de reflejos osteotendinosos rotulianos y $\mathrm{Ba}$ binsky bilateral.

En la Rx de columna dorsal y cervical así como en la Tomografía Axial Computerizada (TAC) torácica (310-2001), se apreciaba una masa mediastínica posterior sugestiva de corresponder con un tumor neurogénico en cara lateral derecha de D4.

Se ingresó en neurocirugía con el diagnóstico de mielopatía aguda con posible compresión medular por tumor a nivel de D4 en mediastino posterior. La Resonancia Magnética Nuclear (RMN) (4-10-2001) (Fig. 1, izda.) se informó como masa epidural que desplazaba el cordón medular hacia adelante a nivel de D4. Ese mismo día se intervino de manera urgente llevándose

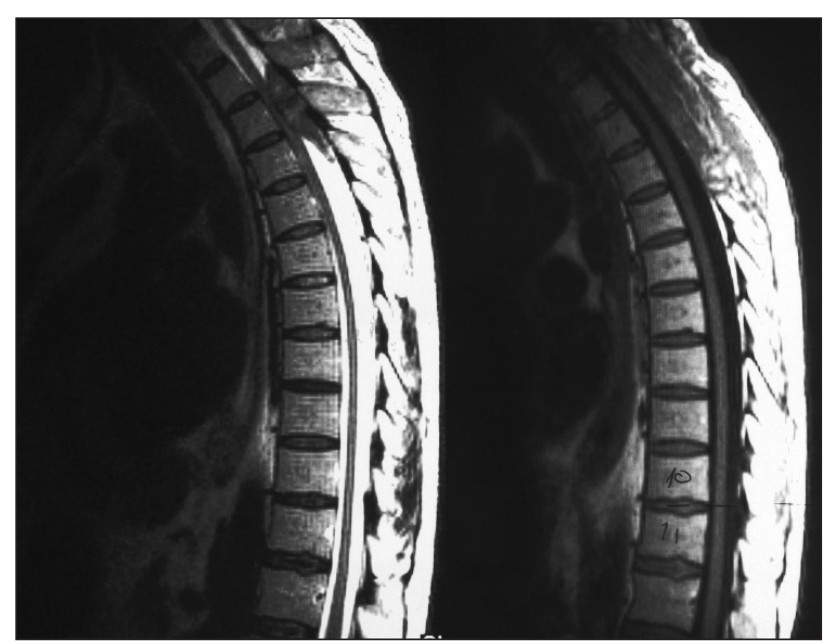

Fig. 1. RMN pre-cirugía a la izda. (04/10/2001) y post-cirugía a la dcha. (10/10/2001) con la correspondiente desaparición de la imagen tumoral. 
a cabo una laminectomía extendida con exéresis tumoral. En el acto quirúrgico se observó una tumoración epidural grisácea negruzca fibrosa adherida a arco dural que se extendía bilateralmente aunque más a la derecha, a través del foramen de conjunción.

La anatomía patológica de la intervención quirúrgica reveló un tejido conectivo infiltrado por melanoma maligno sin infiltración de fragmentos óseos pero sí de los bordes. Sin embargo, no era posible determinar si las lesiones eran metastásicas o primarias originadas en meninges.

El postoperatorio inmediato transcurrió sin incidentes recuperando la hemiplejia izda. a 4/5. En la RMN postintervención (10-10-01) (Fig. 1, dcha). se observaban dos restos tumorales de morfología nodular, uno de ellos en espacio paravertebral derecho y el otro un poco posterior. El estudio de los servicios de dermatología y oftalmología fue negativo.

Se descartó la toracotomía como primera medida terapéutica, y fue trasladado a Oncología Radioterápica para comenzar tratamiento complementario. Se realizó simulación y planificación 3-D e inició dicho tratamiento el día 8 de noviembre de 2001 mediante 2 campos AP y PA y 2 campos oblicuos a $200 \mathrm{cGy} / \mathrm{se}^{-}$ sión hasta completar 48 Gy en la isodosis del 100\%, sobre los cuerpos vertebrales D2 hasta D5 inclusive. En la RMN posterior a este tratamiento $(15 / 01 / 02)$ se observaron de 2 nódulos en relación con restos tumorales ya conocidos, que no habían variado con respecto a estudio previo del 10-10-01.

En febrero de 2002 el paciente fue valorado por el Servicio de Oncología Médica y se solicitó una PET para valorar la persistencia o no de la enfermedad local, demostrar la presencia o ausencia de enfermedad a distancia e intentar localizar un posible tumor primario. Dicha prueba (05-05-02) fue informada con captación a nivel de dos segmentos dorsales altos y región postmedial del parénquima pulmonar (tejido de granulación vs lesión primaria) (Fig. 2).

El día 06-03-02 se realizó intervención quirúrgica mediante toracotomía derecha con la extirpación de masa tumoral, completando los neurocirujanos la resección por estar la masa íntimamente adherida a la duramadre. El informe de anatomía patológica refería la existencia de un melanoma maligno que constituía un nódulo tumoral de $2.5 \mathrm{~cm}$ no encapsulado que infiltraba tejidos blandos, conectivo, adiposo e intraneural en grandes troncos nerviosos. Microscópicamente la proliferación tumoral contactaba con el borde quirúrgico externo del fragmento.

El paciente aceptó el tratamiento que se le propuso con IFN alfa- $2 b$ a dosis de 20 mill. U iv./día $\times 5$ días durante 4 semanas y posteriormente con 10 mill.U

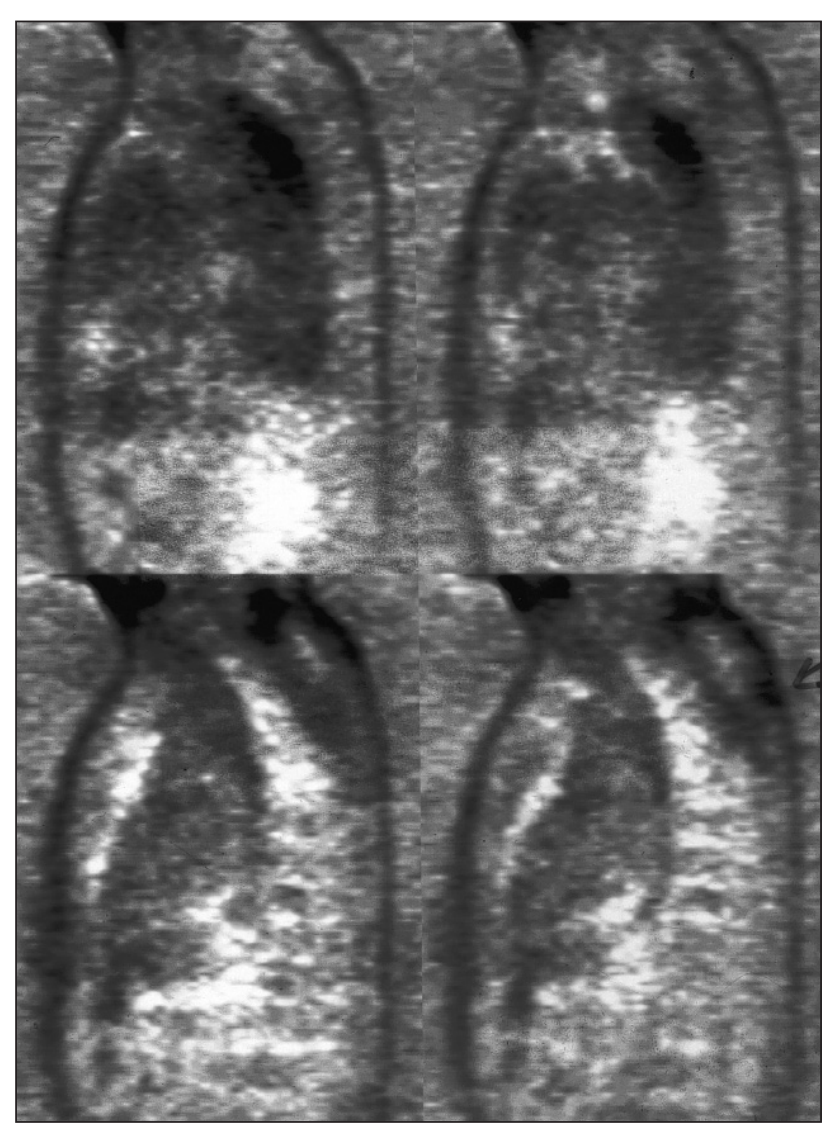

Fig. 2. Imágenes correspondientes a PET (05/05/2002) con persistencia de captación a nivel de dos segmentos dorsales altos y región postmedial del parénquima pulmonar (tejido de granulación vs lesión primaria).

$\mathrm{sc} / 3$ veces por semana hasta completar 52 semanas. El tratamiento fue bien tolerado salvo por algún episodio de neutropenia que obligó a retrasar la administración del mismo. En la RMN del 20/10/02 se observaban cambios postquirúrgicos que habían disminuido con respecto a RMN previas, sin evidencia de tumor ni de compresión medular en la actualidad.

\section{Discusión}

A pesar de que el sistema nervioso central pueda albergar una amplia variedad de neoplasias melanocíticas, incluidas el melanoma maligno metastásico y las relativamente benignas, es muy poca la literatura existente en relación a estas entidades.

En términos generales los tumores pueden afectar a individuos de cualquier edad y pueden aparecer bien como proliferaciones difusas o bien como discretas masas, siendo más frecuentes en la base del cerebro y en la región cervical alta por la habitual localización 


\section{Díaz de Corcuera Frutos y cols.}

de melanocitos en estas zonas. Microscópicamente pueden variar desde lesiones únicas bien diferenciadas, melanocitomas, a proliferaciones malignas para las cuales se aplica el término de melanoma maligno.

Para la diferenciación de ambas entidades es necesario, además de las características microscópicas, tener en cuenta la clínica, la topografía, el propio tumor... entre otras razones, porque los melanomas malignos del sistema nervioso central varían en las características citológicas mucho más que en otros lugares. En ocasiones puede resultar muy dificultoso saber si el tumor está mejor clasificado como melanocitoma o melanoma. Esto se refleja en los artículos de series de melanomas en las que no se intenta distinguir entre estas entidades, por lo que muy poca información está disponible sobre sus características histológicas que son de gran importancia pronóstica'. Por este mismo motivo, la experiencia con este tipo de lesiones es muy escasa y prácticamente nula la existencia de artículos en relación a las mismas, por lo que no disponemos de una bibliografía más amplia que acompañe a nuestro particular caso.

En relación al tratamiento, la cirugía es de elección para la enfermedad localizada mientras que los pacientes con alto riesgo de desarrollar metástasis (estadio IIb-III) pueden beneficiarse del tratamiento complementario con Interferón Alfa-2b (IFNa). La resección de un número limitado de metástasis puede ocasionar un beneficio duradero; sin embargo existen otros casos en los que es preciso la administración de un tratamiento sistémico con citotóxicos con o sin inmunoterapia asociada.

Los quimioterápicos con actividad antitumoral en melanomas metastásicos son entre otros la Dacarbacina (DTIC), los análogos del platino, las nitrosureas y los alcaloides de la vinca. Concretamente el DTIC es considerado el más activo de los agentes con una respuesta alrededor del $20 \%$, con una mediana de duración de 6 meses y una aceptable buena tolerancia, siendo los efectos secundarios más notables las nauseas y los vómitos. La temozolamida es un análogo del DTIC que puede ser absorbido oralmente y con una buena penetración en el SNC, aunque los datos obtenidos en comparación con el DTIC no son lo suficientemente convincentes como para que haya sido aprobado por la FDA (United States Food and Drug Administration) para su uso en el tratamiento del melanoma mestastásico.

La combinación de diferentes agentes ha conseguido en ensayos fase II respuestas de hasta el 30-50\%. Por ejemplo, el régimen CVD (Cisplatino, Vinblastina y DTIC) produjo en algunas series respuestas del $40 \%$ con una mediana de duración de 9 meses, sin embar- go en una reciente puesta al día del ensayo que comparaba el CVD vs DTIC como agente único, no se encontraron diferencias en la tasa de respuesta o en la supervivencia.

Otro esquema que en ensayos inicialmente presentó unas tasas de respuesta de hasta el $46 \%$ fue el régimen de Dartmouth o CDBT (Cisplatino, DTIC, Carmustina y Tamoxifeno). Sin embargo estos datos no han podido ser confirmados con posterioridad y actualmente no existe evidencia de que la combinación frente al DTIC sea mejor. Existen otras posibilidades que están siendo evaluadas: DTIC y tamoxifeno, temozolamida y docetaxel, la bioquimioterapia...

Concretamente y en relación al papel de la inmunoterapia en el melanoma, dos ensayos randomizados ECOG $1684^{2}$ y ECOG $1694^{4}$ han demostrado su beneficio en la adyuvancia de los tumores en estadio llbIII, resecados. En el ensayo de la Eastern Cooperative Oncology Group (ECOG), E-1684 que comparaba el IFNa a altas dosis vs. observación, se comprobó una diferencia en la supervivencia global estadísticamente significativa a favor del tratamiento activo. Posteriormente estos datos se confirmaron en el ensayo E-1694 que comparaba el IFNa a altas dosis con la vacuna GM2. Sin embargo, aunque este beneficio ha sido aceptado por la FDA, el impacto del IFNa en la supervivencia ha sido cuestionado por el costo y la toxicidad del régimen y principalmente por la ausencia de beneficio en la supervivencia en otro ensayo, el E$1690^{3,7}$.

Asimismo, una revisión sistemática de 9 ensayos controlados randomizados ${ }^{5}$ del tratamiento del melanoma con IFNa observó que ningún ensayo, incluidos el ECOG 1684 y ECOG 1694, mostraba significación estadística para la supervivencia global.

Por ello, algunos de los principales objetivos de los ensayos con IFNa en adyuvancia continúan siendo determinar si el tratamiento mejora la supervivencia global, cuál es la dosis adecuada, la duración del mismo y la toxicidad tolerable, que seguirán sin responderse mientras no se conozcan con claridad los mecanismos de actuación antitumoral del IFNa ${ }^{6}$. Actualmente están en marcha diferentes ensayos que intentan responder a éstas y otras preguntas.

La administración de radioterapia en el melanoma tiene varias indicaciones: el tratamiento primario de algunas lesiones, el tratamiento de la enfermedad micro o macroscópica residual post-cirugía y el tratamiento paliativo de síntomas ocasionados principalmente por la diseminación de la enfermedad. Existen también otros ensayos en marcha para evaluar su papel en adyuvancia, como por ejemplo, en la enfermedad ganglionar extracapsular. 


\section{Agradecimientos}

Agradecer a la Dra. Carmen Lobo y a la Dra. Irune Ruiz, especialistas en Anatomía Patológica así como al Dr. Gurutz Garmendia, especialista de Radiología del Hospital Donostia, la aportación y valoración de las pruebas diagnósticas realizadas en este caso.

\section{Bibliografía}

1. Atlas of Tumoral Pathology. Tumors of the Central Nervous System. Peter C, Burger MD, Bernd W, Scheithauer MD. Published by the Armed Forces. Institute of Pathology. Washington D.C. 1994.

2. Kirkwood JM, Strawderman MH, Ernstoff MS, Smith TJ, Borden EC, Blum RH. Interferon alfa- $2 b$ adjuvant therapy of high risk resected cutaneus melanoma: the Eastern Cooperative Group Trial EST 1684. J Clin Oncol 1996; 14:7-17.

3. Kirkwood JM, Ibrahim JG, Sondak VK, Richards J, Flaherty LE, Ernstoff MS, Smith TJ, Rao UNM, Steele M, Blum
H. High and Low Dose Interferon alfa-2b in High Risk Melanoma: First Analysis of Intergroup Trial E1690/ S9111/C9190: J Clin Oncol 2000; 18: 2444-58.

4. Kirkwood JM, Ibrahim JG, Sosman JA, Sondak VK, Agarwala SS, Ernstoff MS, Rao U. High Dose Interferon alfa 2-b significantly prolongs relapse-free and overall survival compared with GM2-KLH/QS-21 vaccine in patients with resected stage IIB-III melanoma: Results of Intrgroup trial 1694/S9512/C509801. J Clin Oncol 2001; 19:2370-80.

5. Lens MB, Dawes M. Interferon alpha Therapy for Malignant Melanoma: a Sistematic Review of Randomized Controlled Trials. J Clin Oncol 2002; 20(7):1818-25.

6. Lynn M, Schuchter A. Cancer Center of the University of Pennsylvania, Philadelphia, PA. Adjuvant Interferon Therapy for Melanoma: High-dose, Low-dose, No-dose, Which dose? J Clin Oncol 2004; 22:1

7. Stergios J Moschos, John M. Kirkwood. University of Pittsburgh Cancer Institute, Pittsburgh, PA Panagiotis A. Konstantinopoulos, State University of New York Upstate Medical Center, Syracuse, NY. Present Status and Future Prospects for Adjuvant Therapy of Melanoma: Time to Build upon the Foundation of High-Dose Interferón Alfa2b. J Clin Oncol 2004; 22:1.
Correspondencia:

Dra. I. Díaz de Corcuera Frutos

Complejo Hospitalario Donostia Hospital Aránzazu Paseo Dr. Beguiristain, s/n E-20080 Donostia (Gipuzkoa) Apartado de Correos 477 E-mail: isukof@yahoo.es 